\title{
Predictive Value of Modified Early Warning Scoring System for Identifying Critical Patients with Malignancy in Emergency Department
}

\author{
Huseyin Aygun, MD1; Suna Eraybar, MD1; Fatma Ozdemir, MD²; Erol Armagan MD, Prof ${ }^{2}$ \\ ${ }^{1}$ Emergency Department, Yuksek Ihtisas Research and Education Hospital, Science Health University, Bursa, Turkey \\ 2 Emergency Department, Uludag University Faculty of Medicine, Bursa, Turkey
}

\begin{abstract}
Background: Identification of critically ill patient is particularly important in the emergency department (ED). The prolonged duration from hospital admission to delivering intensive care service is related to increased mortality. The aim of this study is to evaluate the effectiveness of Modified Early Warning Score (MEWS) for identifying critical patients with malignancy in ED settings. Methods: We evaluated patients with malignancy who were admitted to our ED of a tertiary university hospital in Turkey over a three-month period. We evaluated MEWS on admission as MEWS 1. After the initial treatment depending on the patients' health status in ED, at 2 hours after admission, we evaluated MEWS again and recorded as MEWS 2. All patients were followed up for 30 days after the initial admission.

Results: Mean age (SD) was 59.2 (13.5) and male/female ratio was 295/206. MEWS1 was higher than MEWS2, (MEWS1: $3.05 \pm$ 3.31, MEWS2: $2.35 \pm 3.17, P<0.001)$. A total of 362 patients $(72.3 \%)$ survived and $139(27.7 \%)$ died within 30 days of initial admission. MEWS1/MEWS2 values for alive and dead patients were $1.66 / 0.87$, and $6.67 / 6.21$, respectively, and the difference was significant $(P<0.001)$. ROC analysis was performed for MEWS 1 ; the area under curve (AUC) for hospitalization was 0.768 (95\% Cl 0.729 to 0.804$)$ and for mortality was 0.900 (95\% Cl 0.870 to 0.924$)$. ROC analysis revealed a cut-off value of 2 for predicting both hospitalization and mortality in these patients. The sensitivity of the presented cut-off was $77.32 \%(72.1 \%-82.0 \%)$ for hospitalization and $76.24 \%(95 \% \mathrm{Cl} 71.5-80.5)$ for mortality; the specificity was $69.52(95 \% \mathrm{Cl} 62.8-75.7)$ for hospitalization and 90.65 (95\% Cl 84.65-94.9) for mortality.

Conclusion: We found in our study that MEWS evaluation for patients with malignancy on admission to ED is predictive of mortality in the subsequent 30 days, and it is a valuable tool for identifying the critical group. Also, AVPU scores alone can predict mortality in patients admitted to ED.

Keywords: Critical patient, Emergency department, Malignancy, MEWS

Cite this article as: Aygun H, Eraybar S, Ozdemir F, Armagan E. Predictive value of modified early warning scoring system for identifying critical patients with malignancy in emergency department. Arch Iran Med. 2020;23(8):536-541. doi: 10.34172/ aim.2020.56.
\end{abstract}

Received: September 13, 2019, Accepted: June 2, 2020, ePublished: August 1, 2020

\section{Introduction}

Emergency departments (ED) are generally the first route of admission to health services in the management of the critical patient. ${ }^{1}$ Previous studies showed increased mortality rates when the time between hospitalization and admission to intensive care unit (ICU) is prolonged. Furthermore, mortality is higher in patients who are admitted to ICU from the clinics compared to ED..$^{2-4}$ Early evaluation and initiation of ICU management for critically ill patients prevent cardiopulmonary arrest and mortality. ${ }^{5,6}$ Many scoring systems are utilized in ED for identifying the critically ill patient. ${ }^{7,8}$ Disease severity classifications developed for use in emergency services provide objective measurable results in determining the appropriate time for intervention, improving patient care quality, proper use of resources, and determination of mortality risk. The Glasgow Coma Scale (GCS) is one of the pioneers of these scoring systems. ${ }^{9}$ Some of the others are Rapid Acute Physiology Score (RAPS), Rapid Emergency Medicine Score (REMS), Charlson Comorbidity Index (CCI), Early Warning Score (EWS), and Modified Early Warning Score (MEWS).

Morgan et al first described EWS in 1997, and it was modified by Stenhouse et al in 1999 as MEWS. ${ }^{10}$ This scoring system evaluates systolic arterial blood pressure, pulse rate, respiratory rate, body temperature, and consciousness (Table 1). An Alert, responding to Voice, responding to Pain, and Unresponsive Score (AVPU) scale is used for evaluation of the consciousness state. It was reported that scores greater than 5 define the patients in a high-risk group for discharge. ${ }^{11}$ Some previous studies also reported that MEWS system has predictive value for mortality, ICU admission, cardiac arrest development, survival and discharge in the 60 days after ED admission. ${ }^{7}$ 
Table 1. Modified Early Warning Score (MEWS)

\begin{tabular}{|c|c|c|c|c|c|c|c|}
\hline Score & 3 & 2 & 1 & $\mathbf{0}$ & 1 & 2 & 3 \\
\hline Systolic arterial pressure & $<70$ & $71-80$ & $81-100$ & 101-199 & - & $>200$ & - \\
\hline Pulse rate & - & $<40$ & $41-50$ & $51-100$ & $101-110$ & $111-129$ & $>130$ \\
\hline Respiration rate & - & $<9$ & - & $9-14$ & $15-20$ & $21-29$ & $>30$ \\
\hline Temperature & - & $<35.0$ & - & $35-38.4$ & - & $>38,5$ & - \\
\hline AVPU & - & - & - & A & V & $\mathrm{P}$ & $U$ \\
\hline
\end{tabular}

AVPU, Alert, responding to Voice, responding to Pain, and Unresponsive Score.

MEWS is also an easy-to-use tool that can be performed by the nurses in bedside assessment and does not require a specialized education. ${ }^{12}$

In this study, we evaluated the predictive value of MEWS in identifying the critical patients with malignancy in ED. We also assessed whether the initial treatment in EDs can change patients' MEWS which can predict mortality or hospitalization.

\section{Materials and Methods}

We prospectively evaluated patients with malignancy who were admitted to the Uludag University Faculty of Medicine, Emergency Department over a three-month period. All patients with known malignancy diagnosis admitted to ED were included in the study regardless of whether their complaint was related to their primary diagnosis or not. Patients under 18 years of age and those with cardiopulmonary arrest on arrival were excluded from the study.

A total of 501 patients with primary diagnosis of malignancy were evaluated. The patients' demographic characteristics, diagnoses, chief complaints on admission as well as their systolic blood pressure, pulse rate, temperature, and respiratory rate were recorded on admission. We also collected data on the AVPU on admission and these parameters were used to calculate MEWS. Vital signs were re-recorded after the initial intervention (oxygen, vascular access, fluid resuscitation, antipyretic treatment, etc) as secondary physiological data. For all patients, MEWS was first calculated on admission as MEWS 1 and again after their initial treatment in ED related to their clinical status at the second hour of admission as MEWS 2. Laboratory results, complete blood count and basic laboratory tests (glucose, urea, creatinine, liver enzymes, electrolyte) were also recorded.

We evaluated especially MEWS 1 and its effect on identifying critically ill patients in ED as a gold standard parameter. We also used MEWS 2 to test if symptomatic treatment in ED had any effects on either initial MEWS or patient survival. To use the MEWS as a triage instrument that promises to predict patient disposition and clinical outcome in EDs, we performed ROC analysis to determine the cut-off values for MEWS 1 that should predict hospitalization and mortality. It has been previously reported that scores greater than 5 define the patients in a high-risk group for discharge.

All patients were followed up for 30 days from their first admission to ED, based on either the hospital records or telephone calls to the patients' family.

The study was approved by the local ethical committee of Uludag University Faculty of Medicine.

\section{Statistical Analysis}

Statistical analyses were performed using SPSS 16.0 (SPSS Inc., IL, USA) software. Descriptive data were shown as means, standard deviations, medians, minimum, maximum, and percentages. Normality analysis of continuous data was performed using the KolmogorovSmirnov test. Wilcoxon signed ranks test and MannWhitney $U$ tests were used for comparing continuous and ordinal data of dependent and independent groups, respectively. Binomial test and chi-square test were used for comparison of categorical data. Pearson correlation analysis was used for the associations between numerical variables. Receiver operator characteristics (ROC) analysis and area under the curve (AUC) were used to determine a cut-off value for MEWS scores.

\section{Results}

A total of 501 patients with a mean age (standard deviation) of 59.2 (13.5) years were evaluated. Patients were between 19 to 93 years of age. The male to female ratio was 295/206 (58.9\% vs. 41.1\%). Overall, 105 patients had a primary diagnosis of hematological malignancy. The most prevalent diagnoses regarding the primary cancer types of the patients are presented in Table 2. According to this table, the prevailing malignancies were lung cancer (15.6\%), colon cancer (7.2\%), and acute myeloid leukemia (AML, 7.0\%). The most frequent chief complaints on admission to ED were dyspnea (16.2\%), deterioration in general health status $(14.2 \%)$, and fever $(12.0 \%)$. Other complaints on admission to ED are presented in Table 3. In the study population, $62.5 \%$ of the patients were still receiving chemotherapy.

Measurements of five physiological parameters of temperature, pulse rate, systolic blood pressure, respiratory rate, and consciousness constituted the MEWS evaluation. According to this scoring system, MEWS1 on first 
Table 2. Most Prevalent Cancer Types in Patients with Malignancies

\begin{tabular}{lcc}
\hline Cancer Site & $\mathbf{N}$ & $\%$ \\
\hline Lung cancer & 78 & $15.6 \%$ \\
\hline Colon cancer & 36 & $7.2 \%$ \\
\hline AML & 35 & $7.0 \%$ \\
\hline Breast cancer & 30 & $6.0 \%$ \\
\hline Gastric cancer & 29 & $5.8 \%$ \\
\hline Pancreas cancer & 20 & $4.0 \%$ \\
\hline Bladder cancer & 18 & $3.6 \%$ \\
\hline Multiple myeloma & 18 & $3.6 \%$ \\
\hline GBM & 16 & $3.2 \%$ \\
\hline CLL & 14 & $2.8 \%$ \\
\hline NHL & 13 & $2.6 \%$ \\
\hline Ovarian cancer & 13 & $2.6 \%$ \\
\hline Prostate cancer & 13 & $2.6 \%$ \\
\hline Lymphoma & 12 & $2.4 \%$ \\
\hline Larynx cancer & 11 & $2.2 \%$ \\
\hline Other sites & 145 & $28.9 \%$ \\
\hline Total & $\mathbf{5 0 1}$ & $\mathbf{1 0 0 . 0} \%$ \\
\hline AML, & 13 & $\mathrm{CLL}$ \\
\hline
\end{tabular}

AML, acute myeloid leukemia; GBM, glioblastoma multiforme; CLL, chronic lymphocytic leukemia; NHL, non-Hodgkin lymphoma.

Table 3. Most Frequent Chief Complaints on Admission to Emergency Department

\begin{tabular}{lcc}
\hline Complaint & N & \% \\
\hline Dyspnea & 81 & $16.2 \%$ \\
\hline Deterioration in general health status & 71 & $14.2 \%$ \\
\hline Fever & 60 & $12.0 \%$ \\
\hline Abdominal pain & 44 & $8.8 \%$ \\
\hline Weakness & 44 & $8.8 \%$ \\
\hline Nausea and vomiting & 23 & $4.6 \%$ \\
\hline Seizure & 13 & $2.6 \%$ \\
\hline Confusion & 13 & $2.6 \%$ \\
\hline Swelling & 10 & $2.0 \%$ \\
\hline Coughing & 9 & $1.8 \%$ \\
\hline Other complaints & 133 & $26.5 \%$ \\
\hline Total & $\mathbf{5 0 1}$ & $\mathbf{1 0 0 . 0} \%$ \\
\hline
\end{tabular}

admission to ED, MEWS2 after first intervention in ED, and their parameters are presented in Table 4. The mean MEWS1/MEWS2 values for surviving and dead patients were 1.66 (SD min-max 0-10)/0.87 (SD min-max 0-9), and 6.67 (SD min-max 0-12)/6.21 (SD min-max 0-15) respectively. There was a statistically significant difference in both MEWS1 and MEWS2 between patients' survival status $(P<0.001)$. The mean MEWS1 values were higher than MEWS2 and the difference was statistically significant (MEWS1: $3.05 \pm 3.31$, MEWS2: $2.35 \pm 3.17$, $P<0.001)$.

Complete blood count and laboratory test results of patients in general study group and comparisons between alive and dead patients are presented in Table 5. According to these values, urea $(P<0.001)$, creatinine $(P<0.001)$, AST $(P<0.001)$, ALT $(P=0.03)$, leukocyte $(P=0.01)$, and neutrophil $(P=0.01)$ levels were significantly higher in dead patients. Hemoglobin $(P<0.001)$ and thrombocyte levels were significantly higher in alive patients. There were no statistically significant differences in glucose $(P=$ $0.32)$, sodium $(P=0.17)$ and potassium $(P=0.23)$ levels. After the evaluation at ED, 178 patients $(35.5 \%)$ were hospitalized, 268 patients (53.5\%) were discharged, and 17 patients (3.4\%) died in ED. Totally, 139 (27.7\%) patients died within the period of 30 days. A total of 20 patients died on the same day of admission; in this group, the mean MEWS 1/MEWS 2 were 9.05/9.95 and there was no statistically significant difference.

The difference between MEWS1 and MEWS2 was assessed in relation to survival status. According to this, this difference was calculated as 1.28 in the surviving, 2.26 in the expired, and 1.61 in all patients. Fifty-four patients with MEWS1 < MEWS2 were found and 16 of them were living, while 38 of them expired. However, no significant difference was found in the statistical analysis $(P=0.553)$.

Among patients receiving chemotherapy, 213 (68.1\%) survived and 100 (31.9\%) died within 30 days. In patients who did not receive chemotherapy, 149 (79.3\%) survived and $39(20.7 \%)$ died within 30 days. A statistically significant difference was found in the survival status of patients based on whether they received chemotherapy $(P<0.001)$.

AVPU scores were also assessed for their relationship with mortality. AVPU1 scores for patient who did not survive $(\mathrm{n}=139)$ were distributed as A: 89 patients $(64 \%)$, V: 28 patients (20.1\%), P: 19 patients (13.7\%), and U: 3 patients (2.2\%). AVPU2 scores for this group of patients

Table 4. MEWS Evaluation on First Admission and after Initial Intervention at Emergency Department

\begin{tabular}{|c|c|c|c|c|c|c|c|c|}
\hline & Fever & Pulse Rate & SBP & Respiratory Rate & AVPU & MEWS & \multirow{2}{*}{$\begin{array}{c}\text { Alive } \\
\text { Mean (SD, Min-Max) }\end{array}$} & \multirow{2}{*}{$\begin{array}{c}\text { Death } \\
\text { Mean (SD, Min-Max) }\end{array}$} \\
\hline & Mean (SD) & Mean (SD) & Mean (SD) & Mean (SD) & $\mathbf{N}$ & Mean (SD, Min-Max) & & \\
\hline \multirow{4}{*}{ MEWS1 } & \multirow{4}{*}{$\begin{array}{l}37.01 \\
(0.94)\end{array}$} & \multirow{4}{*}{$\begin{array}{c}96.93 \\
(23.86)\end{array}$} & \multirow{4}{*}{$\begin{array}{c}114.6 \\
(22.85)\end{array}$} & \multirow{4}{*}{19.08 (9.94) } & A: 431 patients & \multirow{4}{*}{$3.05(3.31,0-12)$} & \multirow{4}{*}{$\begin{array}{c}1.66 \\
(0-10)\end{array}$} & \multirow{4}{*}{$\begin{array}{c}6.67 \\
(0-12)\end{array}$} \\
\hline & & & & & V: 43 patients & & & \\
\hline & & & & & P: 23 patients & & & \\
\hline & & & & & U: 4 patients & & & \\
\hline \multirow{4}{*}{ MEWS2 } & \multirow{4}{*}{$\begin{array}{l}36.80 \\
(0.65)\end{array}$} & \multirow{4}{*}{$\begin{array}{l}91.50 \\
(21.76)\end{array}$} & \multirow{4}{*}{$\begin{array}{l}110.60 \\
(17.04)\end{array}$} & \multirow{4}{*}{$18.22(9.42)$} & A: 431 patients & & \multirow{4}{*}{$\begin{array}{l}0.87 \\
(0-9)\end{array}$} & \multirow{4}{*}{$\begin{array}{c}6.21 \\
(0-15)\end{array}$} \\
\hline & & & & & V: 30 patients & & & \\
\hline & & & & & P: 19 patients & $2.35(3.17,0-15)$ & & \\
\hline & & & & & U: 19 patients & & & \\
\hline
\end{tabular}

SBP, systolic blood pressure; MEWS, Modified Early Warning Score; AVPU, Alert, responding to Voice, responding to Pain, and Unresponsive Score. 
Table 5. Hematological Parameters of Patients According to their Survival Status

\begin{tabular}{|c|c|c|c|c|c|c|c|}
\hline & \multicolumn{2}{|c|}{ All Patients } & \multicolumn{2}{|c|}{ Surviving Patients $(n=347)$} & \multicolumn{2}{|c|}{ Dead Patients $(n=122)$} & \multirow{2}{*}{$\boldsymbol{P}$} \\
\hline & Mean & SD & Mean & SD & Mean & SD & \\
\hline Glucose & 135.03 & 67.51 & 135.60 & 67.03 & 133.54 & 68.96 & 0.32 \\
\hline Urea & 52.15 & 37.13 & 44.15 & 27.59 & 72.99 & 48.98 & $<0.001$ \\
\hline Creatinine & 1.13 & 0.94 & 0.99 & 0.73 & 1.50 & 1.28 & $<0.001$ \\
\hline Aspartate transaminase & 40.95 & 75.68 & 31.19 & 37.01 & 66.35 & 127.55 & $<0.001$ \\
\hline Alanine aminotransferase & 32.37 & 51.24 & 27.56 & 29.89 & 44.91 & 83.40 & 0.03 \\
\hline Sodium & 134.91 & 5.23 & 135.01 & 4.99 & 134.64 & 5.83 & 0.17 \\
\hline Potassium & 4.13 & 0.72 & 4.08 & 0.60 & 4.26 & 0.96 & 0.23 \\
\hline Leukocyte & 16.76 & 43.79 & 13.07 & 34.33 & 26.36 & 61.13 & 0.01 \\
\hline Neutrophil & 7.55 & 8.22 & 6.83 & 6.63 & 9.59 & 11.38 & 0.01 \\
\hline Hemoglobin & 10.41 & 2.50 & 10.59 & 2.58 & 9.96 & 2.22 & $<0.001$ \\
\hline Thrombocyte & 244.62 & 175.59 & 261.60 & 172.28 & 200.41 & 177.04 & $<0.001$ \\
\hline
\end{tabular}

were A: 81 patients (58.3\%), V: 23 patients (16.5\%), and U: 15 patients (10.8\%). There was a statistically significant difference between AVPU1 and AVPU2 scores $(P<0.001)$.

We performed ROC analysis to determine the cut-off values for MEWS 1 that should predict hospitalization and mortality in this group of patients. According to sensitivity and specificity of MEWS 1 values at different levels, a cut-off value of 2 was determined for predicting both hospitalization and mortality. The value of AUC was 0.768 (95\% CI 0.729-0.804) for MEWS-hospitalization ROC curve, and 0.900 (95\% CI 0.870-0.924) for MEWSmortality ROC curve. Sensitivity and specificity values were $77.32 \%$ (95\% CI 72.1-82.0) and 69.52\% (95\% CI 62.8-75.7) for hospitalization-MEWS scores, and 76.24\% (95\% CI 71.5-80.5) and 90.65\% (95\% CI 84.5-94.9) for mortality-MEWS scores (Table 6). According to these values, we concluded that a cut-off value of 2 can be

Table 6. Sensitivity and Specificity Values of MEWS 1 Values for Hospitalization and Mortality

\begin{tabular}{lcccc}
\hline & \multicolumn{2}{c}{ Hospitalization } & \multicolumn{2}{c}{ Mortality } \\
\cline { 2 - 5 } MEWS & Sensitivity & Specificity & Sensitivity & Specificity \\
\hline$<0$ & 0 & 100 & 0 & 100 \\
$\leq 0$ & 39.52 & 89.52 & 36.74 & 97.12 \\
$\leq 1$ & 64.95 & 76.19 & 64.09 & 94.96 \\
$\leq 2 *$ & 77.32 & 69.52 & 76.24 & 90.65 \\
$\leq 3$ & 82.47 & 57.14 & 83.15 & 79.14 \\
$\leq 4$ & 87.63 & 47.62 & 89.78 & 71.22 \\
$\leq 5$ & 90.03 & 39.05 & 92.82 & 61.15 \\
$\leq 6$ & 93.13 & 32.86 & 96.13 & 53.96 \\
$\leq 7$ & 94.5 & 27.14 & 97.24 & 45.32 \\
$\leq 8$ & 96.22 & 20.48 & 98.62 & 35.25 \\
$\leq 9$ & 97.59 & 11.43 & 99.45 & 20.86 \\
$\leq 10$ & 98.63 & 6.67 & 100 & 12.95 \\
$\leq 11$ & 99.31 & 1.9 & 100 & 4.32 \\
$\leq 12$ & 100 & 0 & 100 & 0 \\
\hline & & & & \\
\hline
\end{tabular}

used for predicting both hospitalization and mortality in patients with hematological or oncological malignancies who are admitted to ED (Figure 1).

\section{Discussion}

EDs have efficient triage systems that can identify critically ill patients, and admission to intensive care units directly from EDs has better outcome than admissions from other departments. ${ }^{7}$ For this reason, some early warning systems have been developed to identify critically ill patients. According to previous reports, physiological abnormalities on initial admission to hospitals are related to increased ICU needs, increased mortality and prolonged hospitalization. ${ }^{14}$ Delayed evaluation of critically ill patients results in increased cardiac arrest and mortality. ${ }^{15-17}$ We found that approximately $28 \%$ of oncological patients admitted to ED died in the following 30 days. So, identification of critical patients becomes more important in EDs.

Moon et al compared the four-year period before and after utilization of MEWS, and found that cardiac arrest and mortality rates decreased significantly after the utilization of this early warning system. ${ }^{18}$ In 2001, Subbe et al conducted a study on 709 patients and found that MEWS can predict mortality, ICU admission, cardiac arrest, survival, and discharge in the 60-day period after hospital admission. ${ }^{11}$ Patel et al studied the efficiency of MEWS for monitoring physiological deterioration in 32,149 trauma patients ${ }^{19}$; they reported that this score did not contribute to a reduction in mortality, but it was an efficient, valuable, and cheap method for patient followup. Unlike this conclusion, Cei et al suggested in their study that MEWS could predict an unfavorable prognosis even with a single measurement. ${ }^{20}$ Likewise, we found in our study that MEWS evaluation on admission to ED is predictive of mortality in the subsequent 30 days, and it is a valuable tool for identifying the critical patient.

We also assessed whether the difference between MEWS1 and MEWS2 can predict mortality or hospitalization, but the difference had no predictive value. However, we 


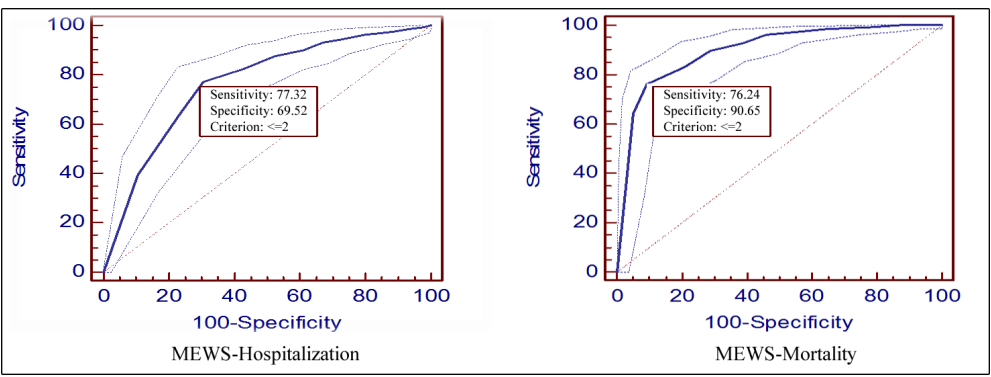

Figure 1. ROC Curves for MEWS 1-Hospitalization and MEWS1-Mortality.

showed that MEWS values on first admission alone were informative of mortality and morbidity.

According to analyses of AVPU scores, we found that these values were correlated with mortality. Of the 27 patients with $\mathrm{V}, \mathrm{P}$, and $\mathrm{U}$ scores, 22 died within 30 days after the admission to ED. This finding was in accordance with the literature. Kelly and colleagues compared the GCS and AVPU scale in a group of patients with toxicological complaints $^{21}$ and found that AVPU was an easy and fast method that can be performed especially by nurses when compared to GCS. This finding was repeated in Akgün's study, ${ }^{22}$ which reported that AVPU scoring was correlated with mortality and hospitalization. According to these studies as well as our findings, we postulate that AVPU scores alone can predict mortality in patients admitted to ED.

We also performed ROC analyses to determine a cutoff value for MEWS that can predict hospitalization and mortality in patients with hematological and oncological malignancies who are admitted to ED. According to our findings, the critical MEWS value was 2, which can predict both hospitalization and mortality in these patients. Fullerton and colleagues ${ }^{23}$ reported that MEWS is a more valuable tool than assessments by paramedics for evaluating an ED patient as critical, and they determined a cut-off of 3 for critical MEWS value. The AUC of this value was 0.799 , and sensitivity and specificity values were $71.1 \%$ and $76.2 \%$, respectively. These values are similar to our results, although there was a difference between the findings of Fullerton and colleagues' study and ours, which involved the MEWS values for patients who died on the same day of admission. They found that MEWS value was 6, and we found that MEWS was 9 in these patients. We think that this difference is related to multisystemic involvement in our terminal stage patients.

In conclusion, patients with malignancy may refer to ED for many reasons and with various complaints. The etiologies underlying the patients' complaints may be reversible in many of the cases. The key point in this regard is timely identification of the critically ill patient for delivering rapid treatment. In this study, we evaluated the MEWS system in a group of ED patients with malignancy and found that this system is valuable for identifying the critical patient. We suggest this scoring system to be used routinely in ED and values of $\geq 2$ have to be considered as critical for patients with malignancy.

\section{Authors' Contribution}

HA conceived the study, designed the study and obtained ethical approval. SE provided statistical advice and analysed the data. FO gave clinical advice on the study subject and methodology. EA supervised the conduct of the study and data related issues.

\section{Conflict of Interest Disclosures}

None.

\section{Ethical Statement}

Uludag University Faculty of Medicine Clinical Research Ethics Committee granted approval for this research.

\section{References}

1. Olsson T, Lind L. Comparison of the rapid emergency medicine score and APACHE II in nonsurgical emergency department patients. Acad Emerg Med. 2003;10(10):1040-8. doi: 10.1111/j.1553-2712.2003.tb00572.x.

2. Goldhill DR, McNarry AF, Hadjianastassiou VG,Tekkis PP. The longer patients are in hospital before Intensive Care admission the higher their mortality. Intensive Care Med. 2004:30(10):1908-13. doi: 10.1007/s00134-004-2386-2 .

3. Goldhill DR, McNarry AF, Mandersloot G, McGinley A. A physiologically-based early warning score for ward patients: the association between score and outcome. Anaesthesia. 2005;60(6):547-53. doi: 10.1111/j.1365-2044.2005.04186.x.

4. 4.Goldhill DR, Sumner A. Outcome of intensive care patients in a group of British intensive care units. Crit Care Med. 1998;26(8):1337-45. doi: 10.1097/00003246-19980800000017

5. Schein RM, Hazday $N$, Pena $M$, Ruben BH, Sprung CL. Clinical antecedents to in-hospital cardiopulmonary arrest. hest. 1990;98(6):1388-92. doi: 10.1378/chest.98.6.1388.

6. Chalfin DB, Trzeciak S, Likourezos A, Baumann BM, Dellinger RP ; DELAY-ED study group. Impact of delayed transfer of critically ill patients from the emergency department to the intensive care unit. Crit Care Med. 2007;35(6):1477-83. doi: 10.1097/01.CCM.0000266585.74905.5A.

7. Grmec S, Gasparovic V. Comparison of APACHE II, MEES and Glasgow Coma scale in patients with nontraumatic coma for prediction of mortality. Acute physiology and chronic health evaluation. Mainz Emergency Evaluation System. Crit Care. 2001:5(1):19-23. doi: 10.1186/cc973.

8. Goodacre S, Turner J, Nicholl J. Prediction of mortality among emergency medical admissions. Emerg Med J. 2006;23(5):3725. doi: 10.1136/emj.2005.028522.

9. Teasdale G, Jennett B. Assessment of coma and impaired consciousness. A practical scale. Lancet. 1974;2(7872):81-4. doi: 10.1016/s0140-6736(74)91639-0.

10. Stenhouse C, Coates S, Tivey M, Allsop P, Parker T. Prospective evaluation of a modified Early Warning Score to aid earlier detection of patients developing critical illness on a general surgical ward. British Journal of Anaesthesia. 2000;84(5):663. 
doi: $10.1093 / b j a / 84.5 .663$.

11. Subbe CP, Kruger M, Rutherford P,Gemmel L. Validation of a modified Early Warning Score in medical admissions. QJM. 2001;94(10):521-6. doi: 10.1093/qjmed/94.10.521.

12. Hennes HJ, Reinhardt T, Otto S, Dick W. The preclinical efficacy of emergency care. A prospective study [in Germany]. Anaesthesist. 1993;42(7):455-61.

13. Armagan E, Yilmaz Y, Olmez OF, Simsek G, Gul CB. Predictive value of the modified Early Warning Score in a Turkish emergency department. Eur J Emerg Med. 2008;15(6):338-40. doi: 10.1097/MEJ.0b013e3283034222.

14. Groarke JD, Gallagher J, Stack J, Aftab A, Dwyer C, McGovern $\mathrm{R}$, Courtney G. Use of an admission early warning score to predict patient morbidity and mortality and treatment success. Emerg Med J. 2008;25(12):803-6. doi: 10.1136/ emj.2007.051425.

15. McQuillan P, Pilkington S, Allan A, Taylor B, Short A, Morgan $\mathrm{G}$, et al. Confidential inquiry into quality of care before admission to intensive care. BMJ. 1998;316(7148):1853-8. doi: $\quad 10.1136 / \mathrm{bmj} .316 .7148 .1853$.

16. Sax FL, Charlson ME. Medical patients at high risk for catastrophic deterioration. Crit Care Med. 1987;15(5):510-5. doi: 10.1097/00003246-198705000-00012.

17. Hargrove J,Nguyen HB. Bench-to-bedside review: outcome predictions for critically ill patients in the emergency department. Crit Care. 2005;9(4):376-83. doi: 10.1186/ cc3518.

18. Moon A, Cosgrove JF, Lea D, Fairs A,Cressey DM. An eight year audit before and after the introduction of modified early warning score (MEWS) charts, of patients admitted to a tertiary referral intensive care unit after CPR. Resuscitation. 2011;82(2):150-4. doi: 10.1016/j.resuscitation.2010.09.480.

19. Patel MS, Jones MA, Jiggins M,Williams SC. Does the use of a "track and trigger" warning system reduce mortality in trauma patients? Injury. 2011;42(12):1455-9. doi: 10.1016/j. injury.2011.05.030.

20. Cei M, Bartolomei C, Mumoli N. In-hospital mortality and morbidity of elderly medical patients can be predicted at admission by the Modified Early Warning Score: a prospective study. Int J Clin Pract. 2009;63(4):591-5. doi: 10.1111/j.17421241.2008.01986.x.

21. Kelly CA, Upex A, Bateman DN. Comparison of consciousness level assessment in the poisoned patient using the alert/ verbal/painful/unresponsive scale and the Glasgow Coma Scale. Ann Emerg Med. 2004;44(2):108-13. doi: 10.1016/j. annemergmed.2004.03.028.

22. Akgün $F$. The efficacies of modified early warning score and mainz emergency evaluation score for prediction of the prognosis in the evaluation of patients attending to TOMC emergency department [thesis]. Malatya: Inonu Universitesi; 2011.

23. Fullerton $\mathrm{JN}$, Price $\mathrm{CL}$, Silvey NE, Brace SJ, Perkins GD. (2012) Is the Modified Early Warning Score (MEWS) superior to clinician judgement in detecting critical illness in the prehospital environment? Resuscitation. 2012;83(5):557-62. doi: 10.1016/j.resuscitation.2012.01.004. 